\title{
Le silence sur la torture pendant la guerre d'Algérie. Analyse d'un corpus de presse française (1957 et 2000)
}

The silence on the torture committed during the war of Algeria. Analysis of a corpus of the French press (1957 and 2000)

El silencio sobre la tortura durante la guerra de Argelia. Análisis de un corpus de prensa francesa (1957 y 2000)

\section{Paola Paissa}

\section{(2) OpenEdition}

\section{Journals}

Édition électronique

URL : https://journals.openedition.org/mots/21476

DOI : $10.4000 /$ mots. 21476

ISSN : 1960-6001

\section{Éditeur}

ENS Éditions

Édition imprimée

Date de publication : 16 décembre 2013

Pagination : $39-54$

ISBN : 978-2-84788-527-9

ISSN : 0243-6450

\section{Référence électronique}

Paola Paissa, « Le silence sur la torture pendant la guerre d'Algérie. Analyse d'un corpus de presse française (1957 et 2000) », Mots. Les langages du politique [En ligne], 103 | 2013, mis en ligne le 16 décembre 2015, consulté le 08 décembre 2022. URL : http://journals.openedition.org/mots/21476 ; DOI : https://doi.org/10.4000/mots. 21476 


\section{Le silence sur la torture pendant la guerre d'Algérie. Analyse d'un corpus de presse française (1957 et 2000)}

La question de la torture en Algérie occupe l'espace discursif public français à deux moments majeurs : au cours de l'année 1957, en plein cœur de la bataille d'Alger; et en 2000, après que la publication du témoignage, dans Le Monde, de Louisette Ighilariz, ancienne militante nationaliste algérienne, relance le débat. À ces deux occasions, le silence acquiert, dans le discours de la presse, une saillance aux facettes multiples, dont cette étude se propose de faire état. En effet, il représente à la fois : 1) un mot qui revient fréquemment dans les articles de journaux et qui, à cause de sa prégnance, est parfois exhibé dans la titraille ; 2) l'enjeu discursif autour duquel pivote la querelle entre ceux qui soutiennent l'impératif moral de dévoiler les méthodes illégales et ceux qui plaident l'opportunité de les taire ; 3) l'arrière-plan doxique auquel il s'agit de s'opposer : le «commode climat de silence» que dénonce le journaliste (chrétien de gauche, membre du PSU et ancien résistant) Claude Bourdet (France Observateur, 11 avril 1957) ou le «silence officiel» que conteste l' «Appel des douze intellectuels pour la condamnation de la torture » (L'Humanité, 31 octobre 2000) indiquent efficacement le mutisme des institutions et l'entreprise d'occultation déployée pour préserver autant que possible, sur la question, le «silence de la doxa " (Bourdieu, 1982, p.154)1. Notre corpus d'analyse se compose de 194 articles tirés du Dossier d'histoire sur la torture durant la guerre d'Algérie, disponible auprès de l'IEP de Lyon². Ce dossier, qui va de 1957 à 2012, rassemble des textes extraits de plusieurs quotidiens et hebdomadaires ayant adopté sur le sujet soit une attitude de polémique plus ou moins virulente envers le gouvernement, soit une position peu ou prou consensuelle3. Nous avons choisi de retenir de ce dossier les articles correspondant aux deux époques indiquées

1. Sur le « secret » entourant la torture et sur la réception des révélations, voir Branche, Thénault, 2000.

2. [http://doc.sciencespo-lyon.fr/Ressources/Documents/DocEnLigne/Algerie/documentstorture.html] (consulté le 30 août 2013).

3. Pour un panorama d'ensemble de la presse à l'époque du conflit, voir Roche, 2007. 
ci-dessus car, à presque cinquante ans de distance l'une de l'autre, elles ont vu se déchaîner de véritables campagnes de presse. Malgré les différences évidentes de contexte historique et médiatique, ces périodes ont en commun de représenter des brèches ouvertes dans le silence doxique auquel nous avons fait référence : dans les deux cas, la multiplication des témoignages, rares et isolés à d'autres moments4, provoque la demande explicite, adressée au pouvoir en place, de constituer une commission d'enquête et d'assumer une position officielle sur l'affaire, face à une opinion publique devenue sensible au problème 5 . Les deux tranches temporelles formant notre corpus sont les suivantes : la première s'étend du 11 avril 1957 au 3 janvier 1958 et la deuxième du 20 juin au 31 décembre 2000. Au printemps 1957, les plaintes portées contre les excès répressifs de l'armée, qui avait assumé des fonctions policières dans le cadre de la loi des «pouvoirs spéciaux» votée en 1956 et du régime militaire d'exception d'Alger, en vigueur depuis janvier 1957, contraignent le gouvernement de mettre sur pied, en avril, une Commission de sauvegarde dont le rapport est rendu public peu avant la fin de l'année6. C'est l'éditorial de Claude Bourdet, «Le silence est de sang» (France Observateur, 11 avril 1957), publié à la veille de la constitution de la Commission, qui marque le début de notre première tranche, alors que le 3 janvier 1958, date du dernier article du dossier commentant la publication du rapport de la Commission, en indique la fin. Au printemps 2000 , le témoignage de Louisette Ighilariz paru dans Le Monde du 20 juin, suivi des aveux des généraux Massu et Aussaresses, provoque la réouverture de la discussion et la parution de nombreux récits des protagonistes de l'époque. En octobre 2000, cette campagne conduit à l' «Appel des douze» et

4. Le quotidien Alger républicain, dirigé par Henri Alleg, a dénoncé, bien avant la guerre, plusieurs cas d'abus policiers; dès 1951, Claude Bourdet publie, dans L'Observateur, «Ya-t-il une Gestapo en Algérie? » et, en 1955, paraît son célèbre «Votre Gestapo d’Algérie ». Quant à l'après-guerre, les amnisties favorisent le silence par voie normative : dans les années soixante-dix surtout, des livres sont publiés, qui ne déclenchent aucun débat (notamment La guerre d'Algérie en quatre tomes, d'Yves Courrière, entre 1968 et 1971; La vraie bataille d'Alger, du général Massu, en 1971, suivi d'un échange de lettres avec Germaine Tillion dans Le Monde; La torture dans la République, de Pierre Vidal-Naquet, en 1972).

5. Raphaëlle Branche et Sylvie Thénault (2000) soulignent l'importance du milieu de provenance des dénonciations. Selon ces historiennes, le scandale de la torture éclate, en 1957, parce que ce sont des chrétiens et des universitaires qui accusent l'armée. En revanche, une affaire comme celle des «torturés d'Oran », ébruitée dans les milieux communistes à l'automne 1956, ne trouve guère d'écoute.

6. De nombreux événements poussent le gouvernement à adopter cette mesure en avril 1957 (voir Branche, 1999) : en février, l'hebdomadaire Témoignage chrétien publie le dossier du soldat Muller; le mois suivant paraît Contre la torture, de Pierre-Henri Simon, qu'Hubert Beuve-Méry présente dans Le Monde ("Sommes-nous les vaincus de Hitler?», 13 mars 1957) ; le 23 mars, la mort suspecte de l'avocat Ali Boumendjel à Alger provoque la démission du doyen de la faculté de droit à Paris; fin mars, le général de Bollardière, qui avait publié le 27 dans L'Express une lettre de soutien à Jean-Jacques Servan-Schreiber, est condamné à soixante jours de forteresse. Une enquête de la Commission internationale contre le régime concentrationnaire (CICRC) a lieu au printemps 1957, à l'initiative de la Croix Rouge internationale, parallèlement à celle de la Commission de sauvegarde, relevant du gouvernement français. 
à la demande, formulée par le PCF, de constituer une commission d'enquête. S'étendant du 20 juin à la fin de l'année 2000 , notre deuxième tranche dessine donc une parabole analogue à celle de la première : l'exigence de transparence et la requête d'une déclaration officielle de la part des autorités font monter la polémique jusqu'à l'automne, avant que la demande de constitution de la Commission ne se désamorce, vers la fin de l'année, comme nous le verrons ci-dessous. Sans prétendre à une exhaustivité qui serait par ailleurs impossible, le corpus formé par les articles du dossier IEP relativement aux deux phases chronologiques décrites, offre des éléments probants pour décrire le rôle et les fonctions du silence sous deux aspects essentiels : au niveau des contenus, il remplit le critère de la représentativité, puisqu'il reproduit la polyphonie des points de vue et l'hétérogénéité des positionnements qui ont nourri la querelle (voirle tableau ci-dessous, pour le détail des journaux dépouillés); au niveau temporel, il retient deux époques délimitées par des pics événementiels précis, correspondant à autant de pics polémiques, qui se répondent en écho d'une période à l'autre. Par ailleurs, le choix de privilégier ces deux moments d'intensification du débat n'exclut pas de tenir compte du réseau discursif qui précède et qui suit les intervalles retenus, les dénonciations d'abus dans le système policier colonial ayant déjà concerné l'Indochine et constituant un fil rouge qui, tout au long $\mathrm{du} 2 \mathrm{O}^{\mathrm{e}}$ siècle, traverse la conscience française comme un fleuve souterrain, émergeant et bouillonnant par moments7. Quant à l'objet de notre étude, c'est en séparant, autant que faire se peut, la notion polyvalente de silence, la fonction indexicale du mot et la complexité relationnelle de l'enjeu discursif, que nous allons organiser cet article. La première partie sera consacrée à définir le statut du silence, relativement au champ notionnel qu'il affiche dans le langage ; la deuxième est destinée à décrire l'environnement lexical et textuel du mot; la troisième est vouée à saisir les coordonnées situationnelles à l'intérieur desquelles le silence, en tant qu'enjeu discursif, est partie prenante8.

\section{Le statut du silence : formes actualisées dans le corpus et orientation axiologique}

Le statut du silence dans le langage est foncièrement paradoxal : signifiant vide, se manifestant par une absence, le silence est doué, dans un «monde préconçu comme expressif» (Bilmes, 1996, p. 135), d'une signification en puissance indéfiniment ouverte et virtuellement infinie, redevable qu'elle est, quasi

7. Les dénonciations d'Andrée Viollis concernant l'Indochine remontent aux années trente (Indochine SOS, 1935); dès 1947, Albert Camus condamne les crimes accomplis à Madagascar et en Algérie. Sur la violence systématique du pouvoir colonial en Algérie et ailleurs, voir Thénault, 2012.

8. Nous réserverons l'emploi de l'italique, pour le mot silence, aux cas où nous ferons strictement référence à l'unité lexicale. 
in toto, du travail d'interprétation. Cette nature intrinsèquement oxymorique, suspendue entre le manque et l'excès, ainsi que l'hypertrophie de la fonction interprétative, expliquent la polysémie et l'ambiguïté de la notion, que la littérature scientifique a souvent soulignées (Puccinelli Orlandi, 1996; Le Breton, 1997). Le trait le plus évident de cette ambiguïté est constitué par l'ambivalence axiologique qui, selon les contextes, peut assumer une orientation positive ou négative. Le silence dont il est question dans cette étude s'inscrit dans une axiologie nettement négative, ce qui s'explique à double titre. Avant tout parce qu'il concerne la violence, situation extrême face à laquelle le choix de se taire n'est jamais ressenti comme légitime. En langue française, un réseau de topiques discursives et comportementales figées relie l'attitude silencieuse au consentement coupable, voire complice (voir Breton, Le Breton, 2009, p. 55) : des topoï argumentatifs (Qui ne dit mot consent; se taire c'est cautionner; se taire face à un crime c'est y contribuer) sont mobilisés dans la protestation contre la couverture des responsabilités sur les sévices, et c'est forte de ces vecteurs éthiques stéréotypés que la dénonciation acquiert souvent la teneur de l'invective : «Tout ce qui tient une plume et qui ne dit mot, consent [...]. L'Histoire dira que la torture a été rétablie en France par ceux qui se sont tus » (L'Express, 14 novembre 1957)9. Deuxièmement, le silence se configure comme une attente frustrée, résultant du hiatus entre un pôle demeurant à outrance taciturne, et un autre estimant indispensable que des paroles à forte valeur performative soient proférées (actes de condamnation, repentance, etc.). La caractérisation négative de ce type de silence découle tant de l'expectative déçue, culminant dans l'expression courante silence assourdissant, attestée dans notre corpus, que du court-circuit qui s'y produit entre les plans du dire et du faire. En effet, il arrive parfois que le silence qu'on déplore, surtout dans la tranche 1957 , soit moins un manque à dire qu'un manque à faire (l'expression fréquente "le silence du gouvernement», par exemple, stigmatise le défaut de publication du rapport de la Commission de sauvegarde; voir ci-dessous). De ce point de vue, la forme de silence constituant notre objet possède, comme le cas précédent, un arrière-fond stéréotypé dans la langue, puisque la conscience épilinguistique populaire opère souvent une superposition entre le dire et le faire, qui affleure dans des locutions comme, par exemple, silence radio, silence électoral, silence des urnes, etc. Dans ces tournures, le silence relève du télescopage des dimensions communicative vs pragmatique et représente une sorte de point de fuite susceptible de prouver, a contrario, que (ne pas) dire c'est (ne pas) faire.

Outre l'orientation axiologique, deux autres caractéristiques contribuent à délimiter notre champ d'analyse. À l'instar des couples oppositifs du manque vs l'excès et du dire vs le faire, d'autres formes que le silence peut assumer,

9. L’article fait partie du célèbre Bloc-notes que François Mauriac a rédigé à partir de 1954 . 
sur le plan langagier général autant que dans les occurrences relevées dans le corpus, se distribuent en correspondance avec quelques binômes antithétiques : au niveau temporel, il peut assumer un statut ponctuel vs continu, alors que, au point de vue social, il peut être individuel vs collectif. L'opposition ponctuel vs continu s'étale suivant un paramètre aspectuel : le silence ponctuel présente une dimension événementielle, variable selon les périodes et les acteurs concernés; par contre, le silence continu s'installe dans le débat en entité morale, à laquelle s'oppose l'instance de la révélation. La dichotomie individuel vs collectif concerne principalement l'expérience des soldats du contingent. Douloureusement individuel lors des événements, le silence des appelés est en réalité un comportement collectif, s'il est vrai, comme l'affirme Raphaëlle Branche, qu'il « soude le groupe des soldats» (Branche, 2001, p.66) dans un lien de honte et de complicité. Les traces de ce mutisme, exiguës dans la tranche 1957, sont foisonnantes en 2000 : en effet, le silence a continué, dans le temps, à hanter les esprits et à garder la nature hybride d'un choix à la fois individuel et collectif, ne parvenant toutefois pas à émerger en tant que phénomène général, faute d'une mémoire apaisée du conflit. En cela, il s'apparente au silence qui touche les victimes et les témoins des drames de l'Histoire, découlant à la fois d'une invalidation de leur parole et de l'indisponibilité de l'auditoire à l'écoute (Steiner, 1969; Dayan-Rosenman, 1998). Enfin, on ne saurait aborder la question du silence au sujet de la guerre d'Algérie sans tenir compte de la censure qui a frappé l'information politique et le langage médiatique à l'époque des faits : en ce sens, le silence représente la conséquence et l'aboutissement d'une stratégie systématique d'obscurcissement, de vidage sémantique et de re-nomination euphémique qui, à partir du refus officiel du signifiant "guerre $»^{10}$, a investi entièrement les désignations relatives aux actes de répression, au point qu'il est vraisemblable, comme l'a suggéré Pierre Vidal-Naquet, que les soldats n'aient pas «mis le mot "torture" sur ce qu'ils savaient qu'on pratiquait» (Le Monde, 28 novembre 2000). Une sorte de silence dans les mots, qui affleure dans plusieurs documents du corpus ${ }^{11}$, enveloppe la construction discursive des événements, et ce n'est peutêtre pas un hasard si la reprise de la discussion se situe en juin 2000, quelques mois à peine après l'acte officiel de nomination de la guerre (Loi n $99-882 \mathrm{du}$ 18 octobre 1999).

10. L'emploi du mot «guerre » n'est toutefois pas absent: le mot s'accompagne soit de caractérisations portant sur la nature de la guerre, entrées dans le jargon militaire après le conflit indochinois (guerre moderne, guerre contre-révolutionnaire, guerre psychologique, etc.), soit de commentaires métalinguistiques indiquant le vide désignatif : "guerre qu'on ne nomme pas ainsi » (Le Figaro, 13 août 1957); «guerre qui n'ose pas dire son nom » (Le Monde, 20 novembre 1957).

11. Les pratiques utilisées pour obtenir le renseignement ont donné lieu à un florilège d'euphémismes: excès, errements, manquements, etc. Sur les désignations concernant la torture, se reporter à Périès, 1997; sur d'autres emplois euphémiques, dans la phase finale de la guerre, voir Paissa, 2012. 


\section{Environnement lexical et textuel du mot silence}

Comme nous l'avons déjà remarqué dans l'introduction, le mot silence a une fréquence élevée dans les articles de presse que nous avons consultés ${ }^{12}$. Le tableau ci-dessous, qui relève d'une lecture intégrale et d'un dépouillement manuel du corpus, montre que $26 \%$ des articles ont recours, une fois ou plus, à ce mot. Le dénombrement a concerné tant la tranche 1957 que 2000 : les premières colonnes de chaque tranche (1 et 5 ) indiquent le nombre d'articles examinés; les colonnes 2 et 6 montrent le total d'occurrences de silence (et, fort rarement, de silencieux) et les colonnes 3 et 7 correspondent au nombre de documents qui en affichent au moins une occurrence. Ce dernier nombre est forcément inférieur au précédent, car le mot silence peut revenir à plusieurs reprises dans le même article : c'est sur ce chiffre minimal que nous avons calculé le pourcentage indiqué ci-dessus, pour éviter que le phénomène des répétitions puisse fausser la donne. En outre, un réseau de mots appartenant à l'isotopie de silence est souvent mis en place : dans notre comptage, nous avons retenu les mots se rapportant à ce lexème ${ }^{13}$, indépendamment de la configuration argumentale qui les caractérise (formes verbales transitives comme silencier, museler, bâillonner, mettre sous le boisseau, ou intransitives, comme se taire, retenir sa langue) et de leur catégorie grammaticale (substantifs comme mutisme, bâillon, non-dit ${ }^{14}$; adjectifs comme muet, laconique). Aux 91 occurrences de silence (colonnes 2 et 6 ), on peut donc ajouter 60 cas d'utilisation d'un mot appartenant à son réseau isotopique (colonnes 4 et 8), ce qui constitue une marque supplémentaire de la prégnance de l'objet. Quant à la distribution des occurrences selon les journaux dépouillés, elle confirme largement que c'est la presse engagée dans la bataille contre la torture qui s'est approprié l'enjeu argumentatif du silence.

L'axiologie dont nous avons expliqué la nature au point précédent est pleinement confirmée par l'observation de l'environnement lexical et textuel du mot. En effet, dans toutes les combinaisons syntagmatiques dans lesquelles silence peut apparaître, les mots cooccurrents possèdent une connotation négative, comme on peut le constater dans les syntagmes que nous avons regroupés cidessous, suivant un critère syntaxique. Dans le groupe verbal, le mot silence couvre généralement la position d'actant objet (ou autre cas-régime); ex :

12. Dans le dossier de l'IEP, nous avons écarté un article écrit en anglais (The Manchester Guardian, 7 mai 1957). En revanche, nous avons retenu La Gazette de Lausanne pour son appartenance à l'aire francophone.

13. Nous nous sommes basée sur les renvois lexicographiques qu'indique le dictionnaire Robert électronique.

14. Ce mot ne figure que dans la tranche 2000 , où il est souvent utilisé comme un synonyme de silence. 


\begin{tabular}{|c|c|c|c|c|c|c|c|c|}
\hline & \multicolumn{4}{|c|}{1957} & \multicolumn{4}{|c|}{2000} \\
\hline & 1 & 2 & 3 & 4 & 5 & 6 & 7 & 8 \\
\hline & $\begin{array}{c}\text { Nombre } \\
\text { total } \\
\text { d'articles }\end{array}$ & $\begin{array}{l}\text { silence } \\
\text { totalité } \\
\text { des occ. }\end{array}$ & $\begin{array}{c}\text { silence } \\
1 \text { occ. } \\
\text { au } \\
\text { moins }\end{array}$ & $\begin{array}{l}\text { Isotopie } \\
\text { de silence }\end{array}$ & $\begin{array}{c}\text { Nombre } \\
\text { total } \\
\text { articles }\end{array}$ & $\begin{array}{l}\text { silence } \\
\text { totalité } \\
\text { des occ. }\end{array}$ & $\begin{array}{c}\text { silence } \\
1 \text { occ. } \\
\text { au } \\
\text { moins }\end{array}$ & $\begin{array}{l}\text { Isotopie } \\
\text { de silence }\end{array}$ \\
\hline Combat & 23 & 4 & 3 & 4 & & & & \\
\hline Demain & 2 & 1 & 1 & 1 & & & & \\
\hline $\begin{array}{l}\text { France } \\
\text { Observateur }\end{array}$ & 10 & 9 & 4 & 5 & & & & \\
\hline La Croix & 5 & & & 1 & 1 & 1 & 1 & \\
\hline $\begin{array}{l}\text { La Gazette } \\
\text { de Lausanne }\end{array}$ & 3 & 3 & 2 & & & & & \\
\hline Le Figaro & 6 & & & & 3 & & & 1 \\
\hline Le Monde & 44 & 12 & 10 & 12 & 42 & 12 & 7 & 7 \\
\hline Le Populaire & 2 & & & 1 & & & & \\
\hline Les Echos & & & & & 3 & & & \\
\hline L'Express & 4 & & & 1 & & & & \\
\hline L'Humanité & 5 & 6 & 3 & 1 & 17 & 17 & 9 & 10 \\
\hline Libération & & & & & 10 & 10 & 6 & 6 \\
\hline $\begin{array}{l}\text { Témoignage } \\
\text { chrétien }\end{array}$ & 7 & 15 & 4 & 9 & & & & \\
\hline AUTRES* & 6 & 1 & 1 & 1 & 1 & & & \\
\hline Total & 117 & 51 & 28 & 36 & 77 & 40 & 23 & 24 \\
\hline
\end{tabular}

\section{Tableau des occurrences}

* Sous la dénomination «AUTRES», nous avons regroupé des journaux dont le dossier IEP ne présente qu'un seul article : L'Aurore, L'Information, L'Intransigeant, La Nation française, Connaissance de l'Algérie. 
reprocher / imposer / rompre le silence; passer sous / réduire au silence, etc., mais il peut aussi assumer la fonction d'actant sujet, donnant lieu à des constructions moins routinières, dont la visée polémique est plus forte : «Laisserez-vous votre silence couvrir cette honte?» (L'Humanité, 10 juillet 1957); "Faut-il penser que le silence est un des moyens de perpétuer une façon de gouverner?» (Le Monde, 2 décembre 2000). Dans le groupe nominal, le mot se retrouve dans deux combinaisons syntagmatiques : la séquence adjectivale, avec épithète postposée ou antéposée, produit des séries négativement marquées, ayant généralement un emploi commun (silence complice; silence gênant; lourd silence; lâche silence, etc.); en revanche, la série silence $+d e+N$, ou $N+d e+$ silence occasionne des rapprochements d'autant plus efficaces, au point de vue argumentatif, qu'ils sont moins prévisibles : «le silence de la peur et de l'intérêt» (La Gazette de Lausanne, 14 avril 1957); « le danger et la trahison du silence» (Le Monde, 17 avril 1957); «l'hypothèque de silence» (Témoignage chrétien, 22 novembre 1957). En outre, l'orientation négative du mot et de son contexte d'usage trouve une confirmation ultérieure dans les réseaux métaphoriques qui relayent la dichotomie silence/parole. À cet égard, un clivage net sépare les articles datant de 1957 et ceux de 2000 : dans les premiers, le couple métaphorique auquel on a le plus fréquemment recours propose l'opposition lumière/obscurité, avec une dévalorisation évidente du pôle de l'obscurité, concernant les acteurs qui, se refusant à parler, s'interdisent également de voir : «l'Armée est aveugle» (L'Express, 19 juillet 1957), les militaires se laissent aller à des «excès aveugles» (Demain, 15 août 1957), les responsables gouvernent «les yeux bandés» (Le Monde, 9 mai 1957) et sont insensibles à "ce qui crève les yeux» (France Observateur, 9 mai 1957); en revanche, dans les textes parus en 2000 , c'est l'antinomie santé/maladie qui prime : empruntant sans doute l'image de la gangrène utilisée par Pierre Vidal-Naquet, sortir du silence équivaut à guérir de la gangrène (L'Humanité, 8 novembre 2000), vider / creverl'abcès (Le Monde, $1^{\text {er }}$ décembre 2000 ; L'Humanité, 23 novembre 2000) ou encore purger la plaie (L'Humanité, 8 décembre 2000).

La centralité et la forte charge affective connectées au mot silence sont enfin tout particulièrement évidentes dans ces segments d'environnement textuel qui, pour leur fonction d'accroche et leur visibilité, ont une importance fondamentale dans la presse écrite, c'est-à-dire la titraille. Notre corpus présente quatre occurrences de silence figurant dans le titre de l'article, une dans un "chapeau » et trois dans les intertitres. Si la plupart des occurrences proposent des tournures lexicalisées, dans lesquelles le mot est pris dans sa dimension événementielle ("Que signifie le silence de M. Billères?», Combat, 15 avril 1957; « M. Delavignette rompt le silence», France Observateur, 11 décembre 1957; «Le silence rompu », L’Humanité, 6 novembre 2000)15, quelques cas de

15. Le «silence de M. Billères» (à l'époque ministre de l’Éducation nationale) se réfère à la 
figure méritent d'être analysés d'un peu plus près. Le premier est constitué par le titre de l'éditorial de Claude Bourdet, «Le silence est de sang (France Observateur, 11 avril 1957), qui a valu au journal d'être saisi, en Algérie et en métropole. Ce titre tire son effet, avant tout, de l'emploi du substantif «sang», fort concret et pathémique, utilisé en position prédicative et préférée à l'attribution adjectivale plus abstraite «le silence est sanglant»; ensuite, de la métalepse efficace qui, faisant l'économie de passages intermédiaires, relie directement le choix de se taire à la conséquence criminelle qu'il produit. Le type de silence pointé dans ce titre est le silence continu, tout comme celui figurant dans le syntagme la «complicité du silence ${ }^{16}$, dans le chapeau qui coiffe l'article «Torture, politique, moralisme» (Témoignage chrétien, 12 avril 1957). Un autre titre, "La sauvegarde du silence » (France Observateur, 10 octobre 1957) vise, par contre, un silence de type ponctuel : néanmoins, l'énoncé possède une forte charge polémique, puisqu'il se rapporte à la réticence du gouvernement à publier le rapport de la Commission (voir ci-dessous), en remplaçant ironiquement les circonstanciels de l'intitulé officiel (Commission de sauvegarde des droits et libertés individuels) par le complément du silence. La même visée critique contre l'inertie du gouvernement, au sujet de l'occultation du rapport, caractérise l'intertitre «Le silence n'est pas une politique» (Témoignage chrétien, 22 novembre 1957). Enfin, un titre de la tranche 2000 présente un changement important de l'acteur visé : le syntagme silence gêné, figurant dans le titre "Les responsables algériens gardent un silence gêné sur la torture» (Le Monde, 2 décembre 2000), ainsi que l'article «Alger se tait sur la torture » (Libération, 4 décembre 2000), dénoncent le mutisme embarrassé des dirigeants politiques de l'autre côté de la Méditerranée, attitude sur laquelle nous reviendrons. Cette brève analyse des titres montre bien que l'observation du mot silence et de son environnement textuel est indissociable de la prise en compte de l'enjeu discursif qu'il revêt. Afin de saisir cet enjeu, s'avère nécessaire un examen plus fin des coordonnées situationnelles et des articulations argumentatives dans le cadre desquelles il se déploie. En revanche, il ne nous sera pas possible ici, faute d'espace, de nous pencher sur le cadre énonciatif des segments retenus : nous signalerons uniquement, dans une note, les fragments relevant du discours rapporté.

démission du doyen de la faculté de droit d'Alger, à la suite de la publication d'une lettre de dénonciation des brutalités (France Observateur, 4 avril 1957); la «rupture du silence» de $M$. Delavignette concerne ses révélations sur le rapport de la Commission. Enfin, le «silence rompu » du premier ministre de la cohabitation Lionel Jospin se rapporte à ses déclarations du 4 novembre 2000 , quand il s'est dit favorable à une commission d'enquête semblable à la Commission Mattéoli sur la spoliation des biens juifs.

16. Les mots «complicité du silence» sont guillemetés dans le texte. 


\section{Le silence en tant qu'enjeu discursif : configuration relationnelle}

Le silence «n'est pas une substance, mais une relation » (Le Breton, 1997, p. 80). C'est cette nature foncièrement relationnelle que nous essaierons de décrire dans ce dernier paragraphe, à travers deux paramètres qui, interagissant entre eux, configurent le silence en tant qu'enjeu : le paramètre actanciel, ayant trait aux acteurs concernés dans les différents cadres situationnels, et le paramètre temporel, scandant les phases de la discussion dans lesquelles le silence s'articule à d'autres thèmes saillants du moment.

Au niveau actantiel, la tranche 1957 se caractérise essentiellement par l'affrontement de deux camps antagonistes : le parti de ceux qui se taisent et celui de ceux qui parlent trop, c'est-à-dire les «défaitistes» (Témoignage chrétien, 12 avril 1957). Les modalités de désignation qu'emprunte la presse la plus favorable à la cause de l'Algérie française à l'encontre des «gêneurs qui os[ai]ent rompre le silence» (Témoignage chrétien, 3 janvier 1958) sont toujours axiologiquement marquées et, partant, sont fort indicatives des différents positionnements des acteurs dans la dispute. Qu'il s'agisse de journalistes peu connus ou, à l'inverse, d'écrivains renommés, tels que François Mauriac ou Pierre-Henri Simon, ou encore d'un compagnon de la Libération comme Claude Bourdet, les désignations auxquelles la presse a recours visent nettement la caricature péjorative : soit on ressuscite le vocabulaire maurassien, en évoquant un fantomatique «complot» des "traîtres» et des «Anti-France» (Témoignage chrétien, 12 avril 1957) ${ }^{17}$, soit on utilise des sobriquets railleurs et dévalorisants (les "doux et dangereux rêveurs", les «belles âmes », les "sacrés chevaliers de la vérité», etc. - L'Intransigeant, 21 avril 1957; L'Information, 30 avril 1957). Dans cette stratégie de la ridiculisation, un indice significatif de la prégnance de l'enjeu du silence est constitué par le renversement isotopique qui se produit. En effet, les «partisans du silence» (France Observateur, 11 avril 1957) soulignent fréquemment le bruit que provoque l'opposition : on se plaint du «tapage des dénonciations » (Combat, 15 avril 1957), on se moque des propos que formulent les « humanitaires les plus bêlants », les «illuminés sophistes» qui se font «bru-

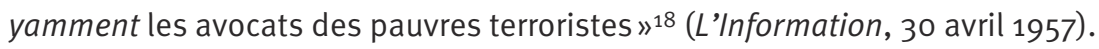
En plus, une sévère distinction est faite entre ceux qui dérangent, mais sont légitimés à prendre la parole, et ceux qui n'en auraient absolument pas le droit. Au premier groupe appartiennent les chrétiens, malgré les déchirements qui traversent leur front; au deuxième, les communistes, dont les dénonciations

17. Ce sont les propos de Louis Terrenoire (journaliste démocrate-chrétien, puis responsable politique «gaulliste de gauche») qui sont rapportés ici.

18. On retrouve l'isotopie de bruit dans la tranche 2000 : par exemple, quand on évoque «le tintamarre de ceux qui s’agitent bruyamment» (René-Victor Pilhes dans Le Monde, 14 décembre 2000). 
sont inacceptables du fait que ceux-ci n'élèvent pas suffisamment la voix contre les horreurs commises par le FLN et qu'ils couvrent d'un silence hypocrite les crimes de Staline (Le Populaire, 20 novembre 1957). Cet essai de «silenciement» d'un groupe politique entier est critiqué, au nom du principe de la responsabilité individuelle, dans L'Express du 6 septembre 1957; néanmoins, il se renouvelle dans la tranche 2000 (Le Figaro, 5 décembre 2000). Quant au paramètre temporel, la période 1957 comprend au moins deux phases (d'avril à juillet et de juillet à décembre) affectant des caractéristiques différentes. Dans la première, c'est le silence continu qui est mis en cause et la bataille que mènent les opposants pour rompre la "spirale du silence »19 est un combat touchant directement aux fondements de la démocratie et à l'ethos de la République. Plusieurs paradigmes mémoriels sont réactivés dans cette querelle : avant tout, il s'agit de la mémoire récente de l'Occupation, de l'époque de l'épuration, marquée par « un silence prudent» (Témoignage chrétien, 3 janvier 1958), et naturellement de la Résistance, bafouée dans la «nouvelle Hitlérie» qu'aurait restaurée en Algérie l'administration Lacoste (France Observateur, 11 avril 1957); deuxièmement, on évoque l'affaire Dreyfus, qui affiche les données circonstancielles et l'épaisseur morale appropriées pour étayer l'opposition fondamentale silence vs vérité (Combat, 11 avril 1957; Témoignage chrétien, 12 avril 1957). Comme à la fin du $19^{e}$ siècle, deuxidées de l'honneur s'affrontent au printemps 1957 : celle de la France démocratique, qui s'affirme en proclamant la vérité, et celle de la Grande Muette qui, sur la base d'une conception de l'honneur patriotique diamétralement opposée ${ }^{20}$, s'efforce de couvrir les responsabilités de son silence proverbial. En outre, les nombreuses saisies de journaux déchaînent des protestations contre le risque d'atteinte à la liberté d'expression : à côté de l'antinomie silence vs vérité, une polarisation silence vs liberté de presse est donc attestée dans plusieurs articles, qui rendent compte d'appels d'écrivains et juristes à l'encontre des tentatives de bâillonner la dissension politique (Combat, 15 avril 1957; L'Humanité, 2 mai 1957). Enfin, la mise sous contrôle gouvernemental de la Commission de sauvegarde engage une discussion sur le statut juridique de cet organisme et sur les tutelles qui pourraient réellement permettre aux témoins de sortir de leur silence (Le Monde, 7 et 9 mai 1957; Combat, 8 mai 1957). Le fait que les valeurs les plus sacrées de la République, ainsi que les garde-fous de la démocratie (pouvoir informatif et judiciaire) soient soumis à une tension si forte, douze ans après la fin du régime de Vichy, explique le curieux phénomène de plagiat langagier qui se produit, le discours des défenseurs du pouvoir semblant emprunter, dans la rhétorique de l'indignation, les mêmes arguments que les «défaitistes», avec

19. Émilie Roche utilise cette notion, empruntée à Élisabeth Noëlle-Neumann, justement pour indiquer la brèche d'avril 1957.

20. C'est la conception ambiguë que décrit si bien Roland Barthes comme composant de la «grammaire africaine» (Barthes, 1970, p. 129). 
une visée symétriquement opposée ${ }^{21}$. Entre autres, c'est justement l'argument du «silence complice» qui subit un renversement de son vecteur polémique, puisque l'accusation la plus fréquente adressée aux «traîtres » est celle de garder un mutisme coupable à l'égard des crimes accomplis par les « rebelles», un grief qui devient d'autant plus violent après le massacre de Melouza du 28 mai : Pierre-Henri Simon, par exemple, est invité à justifier «le singulier silence des bonnes âmes du progressisme sur les atrocités systématiques du FLN » ( $L a$ Nation française, 5 juin 1957) ${ }^{22}$. Le deuxième segment de la tranche 1957 est caractérisé, en revanche, par des discours portant sur un silence de type ponctuel : il concerne le «silence du gouvernement» (Témoignage chrétien, $13 \mathrm{sep}$ tembre; 22 novembre 1957), qui met sous le boisseau le rapport que la Commission avait déposé dès la fin juillet. Le geste éclatant du quotidien Le Monde, qui rend public ce document le 14 décembre, grâce à l'exemplaire reçu par le membre démissionnaire Delavignette, et contraint le gouvernement à une publication précipitée, a fait parler d'un « pouvoir de remplacement » de la presse et d'un «petit coup d'État» (Témoignage chrétien, 20 décembre 1957 et 3 janvier 1958). Les articles condamnant ce silence d'État enchevêtrent les dimensions du dire et du faire, comme nous l'avions remarqué dans le premier paragraphe. Toutefois, la complexité et l'ambivalence de l'enjeu du silence sont, de ce point de vue, particulièrement manifestes : si la récrimination contre l'occultation de faits qui, de toute manière, étaient désormais connus ${ }^{23}$, tend à assimiler le nondire au non-faire, les deux domaines peuvent aussi être ostensiblement séparés dans le discours de dénonciation, pour démasquer l'hypocrisie du pouvoir, qui choisit de châtier le dire plutôt que le faire ${ }^{24}$.

Quant à la période 2000, l'enjeu discursif du silence est encore plus composite, parce qu'il articule constamment les dimensions ponctuel vs continu et individuel vs collectif. Après la publication de «l'Appel des douze pour la condamnation de la torture» (L'Humanité, 31 octobre 2000) et les étonnantes déclarations des généraux Massu et Aussaresses (Le Monde, 22 juin ; 23 novembre 2000), s’affermit une "gigantesque envie de vérité », selon les mots de Vidal-Naquet, «comme si l'affaire Dreyfus surgissait soudainement, cinquante ans après avoir

21. L'hypocrisie qui permet que les uns «tiennent le langage des autres» est explicitement dénoncée dans l'article «La célèbre Jeanne d’Arc » (Le Monde, 5 juillet 1957), défiant résolument l'aumônier parachutiste de s'appuyer sur la mémoire de Jeanne d'Arc pour justifier la torture (Le Monde, 30 juin 1957).

22. Le journal rapporte les mots prononcés par Louis Terrenoire dans un débat avec Pierre-Henri Simon, auteur de Contre la torture (1957).

23. Des interviews des membres de la Commission ont été publiées à partir de fin juillet. La Commission internationale contre le régime concentrationnaire (CICRC) a rendu public son rapport fin juin. En outre, la campagne en faveur de Djamila Bouhired fournit, à partir de juillet, des confirmations ultérieures sur les méthodes des interrogatoires.

24. L'accusation adressée au gouvernement de condamner la parole qui a rendu publiques certaines actions, au lieu de condamner les actions elles-mêmes, est formulée en termes explicites dans l'article de Claude Bourdet du 11 avril 1957. 
été vécue dans l'étouffement» (Le Monde, 28 novembre 2000). Cependant, l'opposition silence vs vérité acquiert, dans cette phase, une portée argumentative bien distincte de celle de 1957. La vérité sur la torture n'étant plus à dévoiler25, les enjeux qui s'affrontent sont autres: d'un côté, c'est le «devoir de mémoire», au nom duquel on demande de « libérer la parole» des victimes ou des anciens d'AFN (L'Humanité, 31 octobre 2000 ; Le Monde, $1^{\text {er }}$ décembre 2000); de l'autre, c'est l'occultation institutionnelle qu'il faut à tout prix faire cesser. Cependant, la discussion s'élargit trop et finit, de ce fait, par s'affaiblir. La proposition du PCF de constituer une commission d'enquête parlementaire provoque des tensions à l'intérieur de la gauche plurielle et d'autres projets voient le jour, qui échoueront également (commission mixte, panel d'historiens, ouverture des archives, mise à la disposition de documents et compatibilité avec les lois d'amnistie). En outre, la demande de «condamnation » contenue dans l'«Appel des douze» se transforme rapidement, dans le discours médiatique, en requête de "repentance». Cette substitution lexicale, contre laquelle réagissent en premier lieu les signataires de l'Appel (L'Humanité, 23 et 27 novembre 2000), n'est pas anodine : l'ambiguïté du mot, son appartenance à un vocabulaire étranger à la tradition laïque française, son extension potentielle à d'autres questions controversées, contribuent, aujourd'hui encore, à faire sentir l'instance de la « repentance» comme irrecevable, ce qui compromet la force perlocutoire de la revendication. L'enjeu silence vs vérité, qui était dominant dans la première phase de la tranche 2000 , se mue bientôt en une antinomie silence vs repentance, et dans cette polarisation le débat s'enlise, puisque les institutions demeurent sourdes. À côté d'un Lionel Jospin à l'attitude quelque peu chancelante, le président Chirac garde, sur tout le dossier, un mutisme qu'aucun appel ne parviendra à briser et qu'on pourrait qualifier de silence d'autorité. Enfin, deux autres formes d'élargissement marquent cette période : d'un côté, l'ouverture de l'arrière-plan historique et géopolitique de référence et, de l'autre, la nette extension de la palette des acteurs concernés. Quant au premier point, on s'interroge sur la nature de la torture, sur son caractère systématique ou épisodique et sur les couvertures qui l'ont rendue possible : dans quelques articles, on parvient à sonder la violence des pratiques policières coloniales avant la guerre d’Algérie (Le Monde, 23 juin et 3 décembre 2000; L'Humanité, 7 décembre 2000). En outre, la « rupture du silence» sur ce sujet est sollicitée, au nom des valeurs républicaines, non plus uniquement dans un cadre franco-français, mais aussi en fonction du droit de la Patrie des droits de l'Homme à prendre position sur nombre de dossiers internationaux, comme l'extradition de Pinochet ou le traitement réservé aux auteurs de graves crimes contre la personne, depuis le Cambodge jusqu'à l'ex-Yougoslavie

25. En 2000 , les dénégations sont pratiquement absentes du débat (sauf peut-être de la part du général Bigeard). En revanche, des justifications au nom de «l'efficacité » sont encore formulées (Le Monde, 18 novembre 2000 ; Le Figaro, 23 novembre 2000). 
(L'Humanité, 7 novembre 2000 ; Le Monde, 2 décembre 2000). Quant au deuxième point, diverses typologies d'acteurs s'ajoutent aux avatars des deux partis principaux. Le «travail de vérité » sur la torture finit par évoluer, dans cette phase, en hypermnésie générale de la guerre : à côté des soldats du contingent, d'autres protagonistes de l'époque sortent du silence, comme les déserteurs, les «insoumis ", les pieds-noirs, les harkis (L'Humanité, 8 et 9 novembre 2000 ; Le Monde, 11 novembre 2000 , etc.), provoquant la cacophonie de la «concurrence des mémoires » qui substitue au silence une rumeur risquant de brouiller également les pistes. En revanche, un autre acteur silencieux entre dans le débat : les responsables politiques algériens, dont le mutisme sur la question est enregistré par la presse française avec surprise, et même une certaine emphase, comme nous l'avons déjà vu à propos de la titraille. C'est à l'adresse des autorités politiques de l'Algérie contemporaine qu'on évoque un «silence sépulcral »26 (Libération, 4 décembre 2000) et qu'on utilise l'oxymore hyperbolique de «silence assourdissant » (Libération, 7 décembre 2000), ou l'expression équivalente de «gêne silencieuse mais néanmoins bruyante» (Le Monde, 27 décembre 2000). En effet, pour ce qui est de l'Algérie actuelle, le silence est également un indice de mauvaise conscience, les dirigeants du pays n'ayant aucun intérêt à soulever la question des pratiques brutales utilisées par l'armée française contre les «martyrs » de la guerre d'Indépendance, vu que les mêmes méthodes sont utilisées contre la dissension dans le présent. Qui plus est, la presse française n'est pas unanime à dénoncer cette attitude algérienne : pour les raisons les plus diverses, qu'analyse bien Hocine Aït Ahmed (rapports de coopération économique, sentiment de culpabilité de la France, etc. : Le Monde, 26 novembre 2000), le gouvernement français, ainsi que plusieurs journaux, jugent plus prudent qu'un couvercle couvre, des deux côtés, l'entière question.

L'opposition clôture vs ouverture, tant au niveau du contexte international qu'à celui du paysage médiatique, constitue le clivage fondamental qui sépare les deux périodes que nous avons examinées. Toutefois, des consonances sont évidentes entre les deux moments du débat, dont la mise en cause du silence est l'épiphénomène le plus significatif. Bien qu'il s'agisse d'un objet évanescent, ne se laissant saisir que par voie indicielle, le silence, décrypté dans ses traces discursives, montre que le non-dit n'est jamais entièrement compatible avec la démocratie. À travers l'articulation des trois perspectives adoptées, nous croyons avoir montré qu'à l'égal de la parole, la non-prise de parole se constitue en événement et s'impose en enjeu de poids dans l'arène politico-médiatique. En particulier, la discussion qui s'est développée autour de la torture, à un moment de crise de la démocratie française comme le fut celui des pouvoirs spéciaux, prouve que le silence occupe une position cruciale entre les instances

26. L'expression est empruntée au quotidien algérien francophone El Watan. 
opposées de la parrhésia, à laquelle tend idéalement le langage démocratique, et l'euphémia, qui caractérise depuis toujours le discours du pouvoir. C'est bien la force de la parrhésia qui a permis, dans une démocratie de longue et solide tradition comme la française, que des voix s'élèvent pour dénoncer les violations de l'État de droit : le silence dont nous nous sommes occupée est donc un "silence fissuré », et c'est le réseau de ces fissures que nous avons pu parcourir.

Cependant, nous sommes consciente aussi que des silences demeurent dans l'histoire qui sont irréductibles à l'analyse, leurs indices étant plus difficiles à cerner. Or, l'histoire coloniale française est pétrie de ces silences (Siblot, 1989) : la torture, pratique emblématique du rapport dominant-dominé, explicitement interdite par les Déclarations des droits de l'homme de 1789 et de 1947 , a occupé le devant de la scène, au moins aux deux époques que nous avons considérées. La réflexion sur ce sujet a dès lors pu fonctionner, pour une partie de l'intelligentsia et de l'opinion publique, comme une sorte de bain purificatoire collectif, mais elle a aussi eu pour rôle paradoxal de faire écran, de dispenser les consciences d'une interrogation sur le sens historique de la « mission » colonisatrice et de provoquer l'élargissement de la «fracture coloniale» (Blanchard, Bancel, Lemaire, 2006). D'autres méfaits ayant ponctué l'épopée glorieuse de la colonisation, ainsi que l'aventure triste de la décolonisation (massacres du Constantinois de 1945 et 1955, bombardement d'Haïphong de 1946, «pacification» malgache de 1947, etc.), ont pu glisser quasiment dans l'oubli (Benot, 1994). Une épaisse chape de silence se profile, en conclusion, derrière le «silence fissuré » que nous avons interrogé ici. C'est alors par les mots d'Alexis Jenny, auteur d'un roman remarquable sur la mémoire déchirée de vingt ans de guerres coloniales et sur ses répercussions dans la société actuelle, que nous allons terminer :

Le prétendu silence autour de la guerre de vingt ans fut un tohu-bohu, une ronde sans fin dont tout le monde se mêlait, et qui tournait, et qui évitait toujours le centre du problème. Si là-bas était chez nous, qui étaient ceux qui vivaient là-bas? Et s'ils vivent ici, qui sont-ils maintenant? Et nous, alors?27

\section{Références}

Barthes Roland, 1970 [1957], Mythologies, Paris, Le Seuil.

Benot Yves, 1994, Massacres coloniaux, Paris, La Découverte.

BILMES Jack, 1996, «Le silence constitué. La vie dans un monde de plénitude de sens», trad. Luc July, Réseaux, vol.XIV, n80, p.129-142.

Blanchard Pascal, BAncel Nicolas, Lemaire Sandrine éd., 2006, La fracture coloniale, Paris, La Découverte.

27. Alexis Jenny, L'art français de la guerre, Paris, Gallimard, 2011, p.600. 
BouRdieu Pierre, 1982, Ce que parlerveut dire, Paris, Fayard.

BRANCHE Raphaëlle, 1999, "La Commission de sauvegarde pendant la guerre d'Algérie.

Chronique d'un échec annoncé», Vingtième Siècle. Revue d'histoire, n61, p.14-29.

BRANCHE Raphaëlle, ThÉnAult Sylvie, 2000, «Le secret sur la torture pendant la guerre d'Algérie», Matériaux pour l'histoire de notre temps, n5 58, p. 57-63.

- 2001, La torture et l'armée pendant la guerre d'Algérie, Paris, Gallimard.

Breton Philippe, Le Breton David, 2009, Le silence et la parole contre les excès de la communication, Strasbourg / Toulouse, Arcanes / Erès (Hypothèses).

Dayan-Rosenman Anny, 1998, "Entendre la voix du témoin », Mots, n56, La shoah: silence... et voix, A. Croll, A. Wieviorka éd., p. 5-14.

Le Breton David, 1997, Du silence, Paris, Métailié (Traversées).

PAISSA Paola, 2012, "La gradualité de l'euphémisme. Analyse d'un corpus de presse à la fin de la guerre d'Algérie », Études pragmatico-discursives sur l'euphémisme, M. Bonhomme, M. de La Torre, A. Horak éd., Francfort-sur-le-Main, Peter Lang, p. 175-192.

PÉRIÈs Gabriel, 1997, «Conditions d'emploi des termes interrogatoire et torture dans le discours militaire pendant la guerre d'Algérie », Mots, n51, p. 41-57.

PucCINelli-OrLANDI Eni, 1996, Les formes du silence. Dans le mouvement du sens, Paris, Éditions des Cendres.

Roche Émilie, 2007, "Étude des discours de presse écrite française sur la violence et la torture pendant la guerre d'Algérie. Le Monde, L'Humanité, Le Figaro, L'Express, France Observateur, 1954-1962 », thèse soutenue à l'Université Lumière Lyon 2, en ligne [http://theses.univ-lyon2.fr/documents/lyon2/2007/roche_e/info], consultée le 30 août 2013.

SıвLot Paul, 1989, «De l'anticolonialisme à l'antiracisme. De silences en contradictions », Mots, $\mathrm{n}^{\circ} 18$, p. 57-74.

STEINER George, 1969, Langage et silence, Paris, Le Seuil.

THÉNAULt Sylvie, 2012, Violence ordinaire dans l'Algérie coloniale, Paris, Odile Jacob. 